\title{
ZY - 3 Satellite Imagery Based Our 1:50000 Topographic Map of Technology and Quality Control
}

\author{
Hong Jiang ${ }^{1}$, Rui Wang ${ }^{1, a}$, Xiaolu Yang ${ }^{1}$ and Renxing Shi ${ }^{1}$ \\ ${ }^{1} 32023$ troops, Dalian, Liaoning, China
}

\begin{abstract}
You should leave $8 \mathrm{~mm}$ of space above the abstract and $10 \mathrm{~mm}$ after the abstract. The heading Abstract should be typed in bold 8,5-point Times. The body of the abstract should be typed in normal 8,5-point Times in a single paragraph, immediately following the heading. The text should be set to 1.15 line spacing. The abstract should be centred across the page, indented $15 \mathrm{~mm}$ from the left and right page margins and justified. It should not normally exceed 200 words.
\end{abstract}

\section{Introduction}

Currently, 90 Kansas, an economically underdeveloped region in the world, belongs to no map region, and the region with 1:50,000 topographic maps in the world only accounts for about $1 / 2^{[1]}$. Restricted by economic, political, natural and traffic factors, such areas cannot be mapped in the field and are collectively referred to as difficult areas. With the continuous development of national political, economic and military aspects, the need for 1:50,000 topographic maps in difficult areas is becoming more and more urgent, and resource 3 satellite (zy-3) image using the non-control point measurement technology has become the preferred data source. Compared with the traditional aerial photography, the zy3 satellite image has the features of low cost, short cycle and strong current situation. Besides, it is not limited by geographical conditions and political and military conditions, and can quickly provide security for emergency.

As the first civil high-resolution surveying and mapping satellite in China, the resource-3 satellite adopts the photogrammetry technology without control points, and some technical indexes of the satellite have reached the similar foreign surveying and mapping satellites. According to the characteristics of resource 3 satellite, this paper designed the technical process of producing 1:50,000 topographic map based on resource 3 satellite image, and conducted beneficial exploration for the future use of resource 3 satellite image in large area surveying topographic map.

\section{Resource 3 satellite}

Resource 3 satellite was successfully launched in taiyuan on January 9, 2012. It is an optical satellite propelled by linear array. Its orbit is a sun-synchronous circular orbit

\footnotetext{
a Corresponding author: author@e-mail.org
}

with an orbital altitude of $505.984 \mathrm{~km}$. The satellite is equipped with a trilinear array panchromatic camera and a multispectral camera. The wavelength range of the front and rear cameras is $0.5-0.8 \mathrm{~mm}$, with a spatial resolution of $3.5 \mathrm{~m}$ and a width of $52 \mathrm{~km}$. The wavelength range of the facing camera is $(0.5 \sim 0.8 \mathrm{~mm})$, with a spatial resolution of $2.1 \mathrm{~m}$ and a width of $51 \mathrm{~km}$. The wavelength range of the satellite's exposure multispectral camera is blue $(0.45 \sim 0.52$ displacement $\mathrm{m})$, green $(0.52 \sim 0.59$ displacement $\mathrm{m})$, red $(0.63 \sim 0.69$ displacement $\mathrm{m})$ and near-infrared $(0.77 \sim 0.89$ displacement $\mathrm{m})$, with spatial resolution of $6 \mathrm{~m}$ and width of $51 \mathrm{~km}^{[2]}$. The ability to simultaneously shoot forward, front and back views, and acquire three-dimensional information of the ground has been successfully applied to the measurement and manufacture of 1:50,000 topographic maps.

\section{Rational function model}

\subsection{Definition of rational function model}

Rational function model is a more generalized expression of sensor imaging model, which is applicable to most sensors. The polynomial expression of the rational function model is as follows:

$$
\begin{aligned}
& \mathrm{S}=\mathrm{Ss} \quad\{\mathrm{P} 1(\mathrm{Xn}, \mathrm{Yn}, \mathrm{Zn}) / \mathrm{P} 2(\mathrm{Xn}, \mathrm{Yn}, \mathrm{Zn})\}+\mathrm{S} 0 \\
& \mathrm{~L}=\mathrm{Ls}\{\mathrm{P} 3(\mathrm{Xn}, \mathrm{Yn}, \mathrm{Zn}) / \mathrm{P} 4(\mathrm{Xn}, \mathrm{Yn}, \mathrm{Zn})\}+\mathrm{L} 0
\end{aligned}
$$

Where, $(\mathrm{Xn}, \mathrm{Yn}, \mathrm{Zn})$ is the coordinate parameter of the principal point, $(\mathrm{S}, \mathrm{L})$ is the coordinate after translation and zooming, (S0, L0) is the standardized parameter, $\mathrm{P} 1(\mathrm{Xn}, \mathrm{Yn}, \mathrm{Zn}), \quad \mathrm{P} 2(\mathrm{Xn}, \mathrm{Yn}, \mathrm{Zn}), \quad \mathrm{P} 3(\mathrm{Xn}, \mathrm{Yn}, \mathrm{Zn})$, $\mathrm{P} 4(\mathrm{Xn}, \mathrm{Yn}, \mathrm{Zn})$ is the polynomial expression of $(\mathrm{Xn}, \mathrm{Yn}, \mathrm{Zn})$. 


\subsection{Features of the rational function model}

Field GPS control points, the cost is high, the cycle is long, cost a lot of manpower, resources and three satellite images can be in the absence of measurement under the condition of control points, using the characteristics of the rational function model, only using own RPC parameter file, you can free net area adjustment calculation, plane precision can reach $15 \mathrm{~m}$, meet the production of 1:50000 topographic map, data accuracy in cost savings and improve the work efficiency under the premise of realize the precise localization of ground targets, it is very significant political, military and economic significance.

\section{The process of making topographic maps of difficult areas}

According to the features of resource 3 satellite image and the technical standard of 1:50,000 topographic map, the measurement process of 1:50,000 topographic map in difficult areas based on resource 3 satellite image is designed (as shown in figure 1). On the premise of the comprehensive analysis of the data of the survey area, the survey area should be reasonably divided according to the characteristics of the regional geomorphology and features. By using the VirtuoZo photogrammetry station of the app company, the original resource 3 satellite image should be preprocessed, encrypted in space and generated DEM automatically according to the design process in FIG. 1. Secondly, geometric correction is carried out for the panchromatic image of resource 3 satellite. Finally, the vector data is collected in the threedimensional environment, and the fusion image resolution is higher than that of the three-line array image. The fusion image is modified by superimposing the vector data and compiled according to the topographic map data standard, and the results are submitted after quality inspection.

\subsection{Preprocessing of the original image}

Due to the interference of weather and atmospheric refractive error, the original resource 3 satellite image has the appearance of thin clouds, mist and shadow. The color contrast between different orbital images is large, which affects the quality of topographic map to some extent. The phenomenon of thin cloud and mist can be processed by PCI Geomatica software to increase texture information of image. For problems such as large shadow and color difference, Photoshop can be used to adjust the contrast and brightness of the original image, and the image information can be enhanced by sharpening tools to make the later three-dimensional sense more obvious, which is conducive to the later collection of vector data in the three-dimensional environment.

\subsection{Air triangulation}

The aerial triangulation of the resource 3 satellite image is divided into three encryption with control point and three encryption without control point. By the control point of the same as the conventional image processing method, here is mainly introduced the control point of the empty three encryption process. Open the VirtuoZo photogrammetry station, firstly import the three-line array image of resource 3 satellite for precision detection, then add the connection point networking to make the free adjustment of the regional network of the image, and finally use the data of related control points of the reference data for precision detection of the results to ensure the quality of the aerial triangulation.

\subsection{Automatic DEM generation}

VirtuoZo software has an automatic DEM module, which can automatically generate DEM according to image matching results. Due to the influence of image color difference, shadow and thin cloud and mist, the matching results and surface situation are contradictory. Therefore, it is necessary to edit the matching results and DEM. When image matching, feature lines should be added to the ridge, saddle and valley, which can greatly improve matching accuracy and reduce DEM error. After automatically generating DEM, the data is set to a regular network, which is superimposed with the stereo image, and DEM data is manually modified to improve DEM accuracy.

\subsection{Create DOM}

The PCI Geomatica software is used to produce the orthophoto image of resource 3 satellite, which can be used for image processing in large quantity, with high absolute precision and edge precision. The specific process is as follows: new project, imported original image, DEM correction, image fusion, image Mosaic, cutting width, uniform light and uniform color, quality inspection, delivery of results. Compared with other similar orthophoto image processing software, PCI Geomatica software has obvious advantages in dealing with over a thousand orthophoto images .

\subsection{D Map}

Stereoscopic mapping is mainly the collection of vector data of ground objects and landforms. It is mainly based on the location of ground objects. The ground objects that can be read on the three-dimensional image of the threeline array can be directly determined by attributes. What needs to be paid attention to is that the location of the ground objects should be accurate, the point ground objects should be cut into the central point of the ground objects, the line ground objects should be collected in the central position of the ground objects, and the surface ground objects should be collected along the boundary of the ground objects and closed. Due to the difficulty of field rendering, we can only rely on the operator to interpret the images indoors. After fusion, the orthophoto image of resource 3 satellite is of high resolution, clear texture and easy to read. It can be used as an auxiliary image for stereo mapping and can greatly improve the quality of image interpretation.

\subsection{Edit topographic map data}

After the vector data collection is completed, it shall be imported into the editing software and edited according to 
the topographic map standard. First, check whether the code is used correctly, whether there is redundant garbage data, and strictly follow the specified data stratification; Then, according to the standard of topographic map data, the topological relation of the data is processed. At last, it makes topographic map publication data, correctly handles the pressure-cover relationship between symbols, keeps the logical consistency between ground objects, and ensures the beauty and style of the map surface.

\section{Suggestions for improving topographic map quality}

\subsection{Reduce the error of air three encryption results}

Because of satellite in the process of filming, orbit offset and oblique photography problems such as the interference, to some extent, affect the quality of three encryption result, this needs in the test area, divided into measurement area should have multiple images of orbit, unify to carry on the adjustment, and according to the difficult areas can't measure the field control points of special circumstances, to meet our under the standard of 1:50000 topographic map, relax appropriately tolerance, to make sure three encryption work. Difficult terrain aerial triangulation lack of or no field measurement control point, can only rely on images bring *. Settlement RPC file, the error caused by human factors had a greater influence on the quality of three encryption, so in establishing a connection point, point try to choose the road intersection or characteristic obvious position, should compare each JingYing repeatedly in the threedimensional environment as point, reduce the human error, to improve the quality of topographic map results.

\subsection{DEM data editing}

The best way to make DEM is to combine automatic image matching with manual interaction. The DEM data generated by automatic image matching is highly accurate when there is no vegetation on the ground or only grassland, and the error is large in the shadow areas of forested areas and mountains, which requires manual editing. In editing, attention should be paid to areas with high buildings, viaducts, Bridges, DAMS and other ground objects. DEM represents surface elevation, not surface elevation. In the construction of topographic map data database, lake, ocean, reservoir and other static surface waters are theoretically regarded as a plane, and the elevation is consistent. If the elevation is inconsistent, it becomes a curved surface, which is wrong. When edge grafting, it is necessary to carefully check whether there are loopholes, and conduct edge grafting in strict accordance with edge precision limit difference, so as to avoid large error, which will affect the quality of DEM results.

\subsection{Orthophoto interpretation}

The resolution of the three-dimensional image of resource 3 satellite is $3.5 \mathrm{~m}$, the smaller independent ground objects and houses are not easy to distinguish, the edge lines of the elements are not clear enough, and they need to be interpreted by other image data. Will be $2.1 \mathrm{~m}$ resolution panchromatic image and $5.8 \mathrm{~m}$ resolution multispectral image after geometric correction, image fusion, the generated orthogonal projection as high resolution, texture clear, obvious characteristics, color is clear, is three linear array stereo images resources satellite no. 3 important indoor interpretation of auxiliary materials, can improve the resources satellite images of indoor interpretation no. 3 quality, so as to further improve the quality of topographic map results.

\subsection{Focus on editing vector data}

When editing and building database data, the layer relationship of the elements should be set as required. The topological relation among points, lines and planes should be correctly handled, and repeated lines, mutual intersection and self-intersection should be eliminated. Correctly input the property and property information of the data, and connect the ground object pointer to the concerned information, so as to avoid the loss of ground object information caused by human. The published data of topographic map is mainly carried out in accordance with the standards of the task design book, the relationship between the elements is reasonably handled, the gland between the symbols is properly handled, and the decoration of the elements outside the outline is well done. In editing, the focus is on the relationship between the elements, the length of the bridge over the double-line river, the Angle at the junction of the river, the upper and lower layer of the overpass and ramp, the flow of the river from the lowest point of the contour line, the compaction relationship between the annotation and the ground objects, the reverse of the wall, the density of the vegetation symbol, and so on.

\section{The conclusion}

Resource 3 satellite adopts the technology of no control point measurement. It can survey difficult areas without the need of field measurement control points, which changes the traditional dependence on foreign WorldView images and fills in the blank of China's civil surveying and mapping satellite, and has a broad prospect of development. Although in terms of image resolution, orientation and similar foreign satellite images there is a gap, but still able to meet the needs of the difficult areas of 1:50000 topographic map of our work, and can be in the investigation of land and resources, environmental protection, geological disaster monitoring, energy exploration, disaster relief and other aspects play an important role in, believe that with the rapid development of computer technology and sensor technology, in the near future will be able to catch up with similar foreign satellite images. 


\section{References}

1. R Wang. Science of surveying and mapping. J. 5-7, (2013)
2. $P$ Wu. Mapping and spatial geographic information. J. 109-113, (2015) 